\title{
Association of fish consumption and dietary intake of marine $n$-3 PUFA with myocardial infarction in a prospective Danish cohort study
}

\author{
Anders Gammelmark ${ }^{1 *}$, Michael S. Nielsen ${ }^{1}$, Christian S. Bork ${ }^{1}$, Søren Lundbye-Christensen ${ }^{1,2}$, \\ Anne Tjønneland ${ }^{3}$, Kim Overvad ${ }^{1,4}$ and Erik B. Schmidt ${ }^{1}$ \\ ${ }^{1}$ Department of Cardiology, Aalborg University Hospital, 9000 Aalborg, Denmark \\ ${ }^{2}$ Unit of Clinical Biostatistics and Bioinformatics, Aalborg University Hospital, 9000 Aalborg, Denmark \\ ${ }^{3}$ Danish Cancer Society Research Center, 2100 Copenhagen, Denmark \\ ${ }^{4}$ Section for Epidemiology, Department of Public Health, Aarbus University, 8000 Aarbus, Denmark \\ (Submitted 23 January 2016 - Final revision received 31 March 2016 - Accepted 5 April 2016)
}

\section{Abstract}

Several studies have investigated the potential benefits of marine $n$-3 PUFA in CVD, generally suggesting a lower risk of CHD. However, recent trials have questioned these results. This study investigated the association of fish consumption with dietary intake of marine $n-3$ PUFA with incident myocardial infarction (MI). In a Danish cohort study, 57053 subjects between 50 and 64 years of age were enrolled from 1993 to 1997. From national registries, we identified all cases of incident MI. Dietary fish consumption was assessed using a semi-quantitative food questionnaire, including twenty-six questions regarding fish intake. In addition, we calculated the intake of total and individual marine $n-3$ PUFA. During a median follow-up of 17.0 years, we identified 3089 cases of incident MI. For both men and women, a high intake of fatty fish was inversely related to incident MI. Thus, when comparing the highest and the lowest quintile of fatty fish intake, we found a $12 \%$ lower relative risk of $\mathrm{MI}$ in men (hazard ratio (HR) $0 \cdot 88 ; 95 \% \mathrm{CI} 0 \cdot 77,1.00)$ and a $22 \%$ lower relative risk in women (HR $0.78 ; 95 \% \mathrm{CI} 0 \cdot 63,0.96)$ after adjustments. For women, similar associations were observed for individual and total marine $n$ - 3 PUFA. In contrast, intake of lean fish was not associated with MI. In conclusion, incident MI was inversely related to a high intake of fatty fish, but not lean fish. However, test for trends across quintiles was not statistically significant. In general, this study supports the view that consumption of fatty fish may protect against MI.

Key words: Myocardial infarction: Cohort studies: Marine $n$-3 PUFA: Fish consumption

The potential benefit of marine $n$-3 PUFA on CHD has been extensively examined in both observational studies and clinical trials, with the majority of studies favouring a beneficial effect of $n-3$ PUFA $^{(1-4)}$.

Thus, several prospective cohort studies have reported an inverse relationship between fish consumption and $\mathrm{CHD}^{(1,5-14)}$. However, other studies have not supported these findings ${ }^{(15,16)}$. Most epidemiological studies have evaluated the dietary intake of fish using questionnaires, but relatively few of these studies have differentiated between different types of fish and their content of marine $n-3$ PUFA. In this study, we evaluated fish intake using detailed and validated FFQ, allowing us to differentiate between consumption of fatty and lean fish, and additionally to calculate the intake of total and individual marine $n-3$ PUFA.

Large secondary prevention trials have been performed evaluating dietary interventions with fish servings or supplementation with fish oil capsules. Although earlier trials such as the diet and reinfarction trial ${ }^{(17)}$, Gruppo Italiano per lo Studio della Sopravvivenza nell'Infarto Miocardico (GISSI)
Prevenzione $^{(18)}$ and Japan EPA Lipid Intervention Study trials ${ }^{(19)}$ have demonstrated a reduction in CHD events following consumption of marine $n-3$ PUFA, more recent trials have not supported these findings ${ }^{(20-22)}$. Controversy therefore still remains regarding the potential benefits from fish consumption and $n$-3 PUFA supplements in the prevention of CHD, and guidelines are currently under review.

In this large Danish cohort study, including 3089 validated cases of incident myocardial infarction (MI), we investigated the association of fish consumption and dietary intake of marine $n-3$ PUFA with incident MI. The present study is one of the largest cohort studies on fish consumption and MI, and notably the study includes a relatively high number of female cases.

\section{Methods}

Study population and design

The Danish Diet, Cancer and Health study is a prospective cohort study, which has been described in detail previously ${ }^{(23)}$.

Abbreviations: DPA, docosapentaenoic acid; ICD; International Classification of Disease; MI; myocardial infarction.

* Corresponding author: A. Gammelmark, email anders.gammelmark@rn.dk 
In brief, 160725 persons aged 50-64 years were invited between December 1993 and May 1997. Inclusion criteria were as follows: born in Denmark, living in the urban areas of Copenhagen and Aarhus and not registered with a cancer diagnosis in the Danish Cancer Registry at the time of invitation. A total of 57053 persons accepted the invitation and were enrolled into the study. Participants registered with a previous MI or cardiac arrest were excluded. If a cancer diagnosis was reported, which was not already recorded in the Cancer Registry at the time of invitation, participants were excluded in line with the intention-to-include criteria. At baseline, each participant filled in a detailed questionnaire on diet, lifestyle, socio-economic status and medical history. Data from dietary questionnaires were analysed in a traditional cohort design including the entire cohort. Participants were followed-up until July 2013. The present study was conducted according to the Helsinki Declaration and approved by the regional Ethics Committees.

\section{Dietary assessment}

At baseline, participants filled in a detailed 192-point, semi-quantitative FFQ, including twenty-six specific questions regarding the intake of fish and food products containing fish. The questionnaire has been validated and described in detail previously $^{(24)}$. In short, participants were asked to estimate their daily intake of foods in natural units such as pieces of fruits, slices of bread and glasses of different drinks. For mixed dishes and meals, sex-specific portion sizes were calculated using data from a calibration study. By multiplying the frequencies of intake by the portion size, the individual average intake in $\mathrm{g} / \mathrm{d}$ of all foods and nutrients was calculated. Different species of fish were categorised as either lean or fatty depending on their content of $n$-3 PUFA, below or above $1 \mathrm{~g} / 100 \mathrm{~g}$, respectively. Fatty fish mainly comprised herring, salmon, trout and mackerel, whereas lean fish comprised mainly plaice, flounder and $\operatorname{cod}^{(7)}$.

The dietary intakes of specific nutrients including total and individual marine n-3 PUFA - EPA, docosapentaenoic acid (DPA) and DHA - were calculated using FoodCalc software (www.ibt.ku.dk/jesper/foodcalc) based on Danish Food Composition Tables.

\section{Outcome assessment}

We identified all participants in the cohort who were registered with an incident diagnosis of MI in the Danish National Patient Registry and/or the Danish Causes of Death Registry, according to the International Classification of Disease (ICD) 8 (410.00410.99) or ICD-10 (I21.0-I21.9) coding, during the study period. Furthermore, all cases of cardiac arrest (ICD-8: 427.27 or ICD-10: I46.0-I46.9) were included if the arrest was considered to be of cardiac origin after validation in each individual case. The Danish National Patient Registry ${ }^{(25)}$ contains information on diagnoses and interventional procedures in relation to all hospital ward admissions since 1977, whereas all visits to outpatient clinics and emergency rooms have been registered since 1995. The Danish Causes of Death Registry contains diagnoses for all deaths since 1943. Patients were registered in both registries in accordance with ICD-8 until 1 January 1995 and subsequently according to ICD-10. An earlier study from our Department validated the MI diagnosis from baseline through 2003 by complete review of all medical records, and found a positive predictive value $>92 \%$ when the diagnoses were obtained from a hospital ward ${ }^{(26)}$. All validated cases of MI from the validation study were readily accepted as cases for the present study. From January 2004 through July 2013, all participants with an incident MI diagnosed from a ward were accepted as cases without further validation. All other diagnoses of incident MI and cardiac arrest were validated by reviewing a complete list of diagnoses and interventional procedures recorded in the Danish National Patient Registry for each potential case. Cases of incident MI were categorised as fatal or non-fatal depending on their vital status $28 \mathrm{~d}$ after the event. Vital status was obtained using the Civil Registration System.

\section{Statistics}

Exposure variables were categorised into quintiles based on the cohort distribution. A test for trend across quintiles was carried out. Furthermore, we also evaluated the exposure variables as continuous variables using restricted cubic splines with three knots.

Measures of association were assessed using Cox proportional hazards multivariate regression models with age as the time axis and delayed entry. Analyses were conducted separately for men and women. Participants were treated as at risk from baseline until either MI, death, emigration or end of follow-up occurred.

To address potential confounding, we adjusted for traditional risk factors of MI (model A2), including smoking habits (never, former or current $(<15,15-25,>25 \mathrm{~g} / \mathrm{d})$ smoker), BMI $\left(\mathrm{kg} / \mathrm{m}^{2}\right)$, waist circumference $(\mathrm{cm})$, physical activity (h/week), alcohol intake $(\mathrm{g} / \mathrm{d}$ ), educational level (basic school, higher education: $1-3$ or $>3$ years) and, for women, menopausal status (pre or postmenopausal). Second, we adjusted for either medical history (model B) or additional dietary covariates (model C) in separate models. Regarding medical history, we adjusted for history of diabetes mellitus (yes/no), hypertension (yes/no) or hypercholesterolaemia (yes/no), including participants on antihypertensive medications or lipid-lowering drug treatment, respectively. Adjustment for additional dietary variables depended on the exposure variable (details specified in the relevant tables). All continuous variables were included in the models as restricted cubic splines with five knots. Potential confounders were selected a priori based on current knowledge of risk factors for MI.

The proportional hazards assumption was checked by visual inspection of $\log -\log$ plots and by evaluation of scaled Schoenfeld residuals, with no significant violations. Estimates with $P$ values (two-tailed) $<0.05$ were considered statistically significant. STATA, version 13.1 (StataCorp. LP) was used for statistical analysis.

\section{Results}

The Diet, Cancer and Health cohort included a total of 57053 participants. From these, an initial 1506 were excluded when 


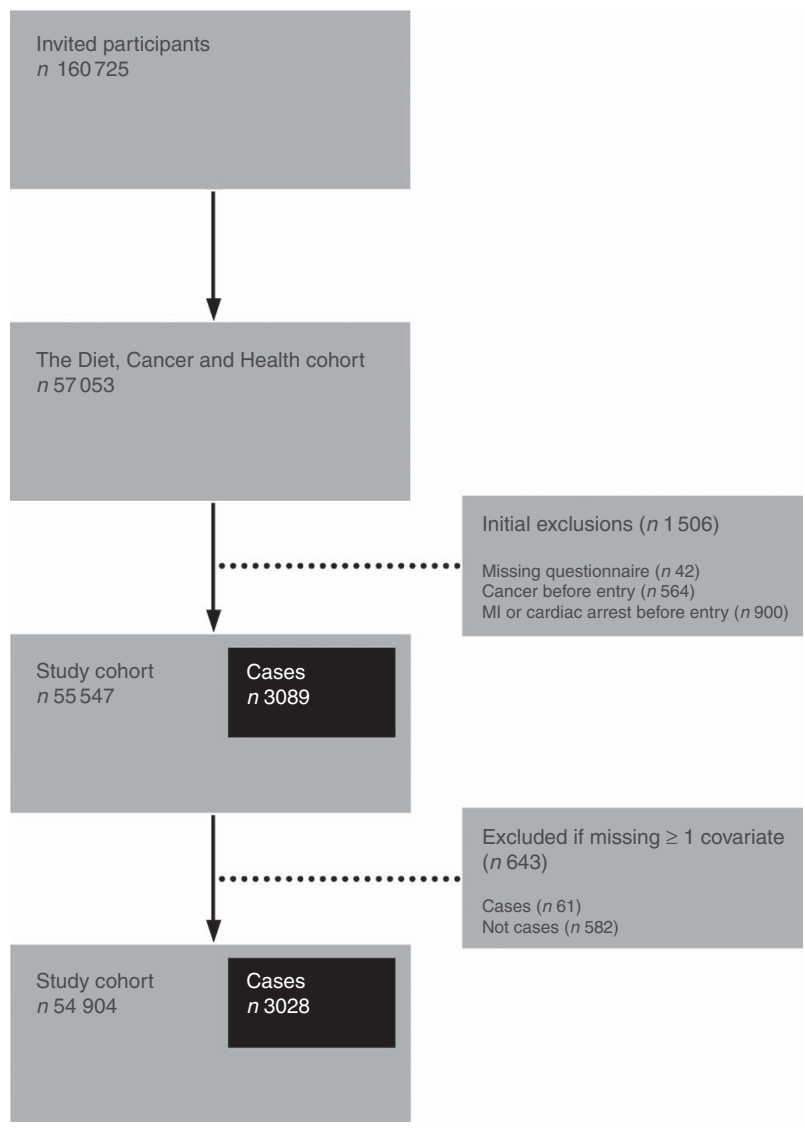

Fig. 1. Flow chart of cohort selection process. MI, myocardial infarction.

baseline questionnaires were missing ( $n$ 42) or when participants were identified with a cancer diagnosis in the Danish National Patient Registry before baseline ( $n$ 564). We also excluded participants with previous MI or cardiac arrest before inclusion ( $n$ 900). In our study population, we identified 3089 cases of incident MI during a median follow-up time of 17.0 years. After case verification, we excluded subjects for whom information on one or more covariates used in the adjusted analyses was missing ( $n$ 643). Thus, 3028 cases were included in the final analyses (Fig. 1).

Baseline risk factors for CHD were generally more prevalent in cohort members who experienced a MI during follow-up ('cases') than in the cohort as a whole (Table 1). Accordingly, we observed a larger proportion of men, higher age, BMI and waist circumference, and a larger proportion of smokers among cases. In contrast, cases had lower weekly physical activity and lower educational level compared with the cohort. With regard to medical history, more cases suffered from hypertension, hypercholesterolaemia and diabetes mellitus. Consumption of fruits and vegetables was lower among cases, whereas we did not find any marked differences between cases and the cohort with regard to fish consumption and use of fish oil supplements. Baseline characteristics were also evaluated by the intake of fatty and lean fish for men and women separately, in order to describe potential confounders. Data are included in the online Supplementary Material (online Supplementary Table S1.2).

\section{Fish consumption and incident myocardial infarction}

The median intake of fatty fish was $15 \cdot 1$ and $12.2 \mathrm{~g} / \mathrm{d}$ for men and women, respectively, whereas the median intake of lean fish was 24.4 and $21.0 \mathrm{~g} / \mathrm{d}$ for men and women, respectively, with minor variations between cases and cohort (Table 1).

In Table 2, we present hazard ratios (HR) for the association between fish consumption and incident MI distributed by quintiles. For men, fatty fish intake was inversely associated with MI in the four higher quintiles compared with the lowest. The association was attenuated by adjustment for traditional risk factors for MI, but the trend remained for the highest quintile compared with the lowest when adjusting for traditional risk factors and medical history (HR 0.88; 95\% CI 0.77 , 1.00). For women, we found an inverse association between the highest quintile of fatty fish consumption and MI, which remained statistically significant after adjustment for traditional risk factors and medical history (HR 0.78; $95 \%$ CI 0.63, 0.96). Adjustment for dietary variables did not affect the measures of association substantially; however, results for model C were not statistically significant. The test for trends across quintiles was not statistically significant in the adjusted models, and a linear dose-response relationship could not be demonstrated. No consistent associations were seen between consumption of lean fish and MI, for neither men nor women.

In addition, fish intakes were evaluated as continuous variables, modulated as restricted cubic splines with three knots, suggesting an inverse trend between intake of fatty fish and MI for both men and women, whereas lean fish was not associated with MI (Fig. 2).

\section{Dietary intake of marine n-3 PUFA and incident myocardial infarction}

We also examined the intakes of individual and total marine $n-3$ PUFA (EPA, DPA and DHA). The median intake of total marine n-3 PUFA was 0.7 and $0.6 \mathrm{~g} / \mathrm{d}$ for men and women, respectively. As expected, intake of fatty fish was highly correlated with the calculated intake of marine $n$-3 PUFA ( $r$ 0.93, $P<0 \cdot 001)$. For men, we observed a trend towards a negative association between EPA, DHA and total marine $n$ - 3 PUFA when comparing the highest and lowest quintiles; however, the associations were modest and not statistically significant for any of the models (Table 3). Results were generally more consistent, towards an inverse association, between marine $n$-3 PUFA and incident MI for women. Accordingly, intake of total and individual marine $n$ - 3 PUFA was inversely associated with MI. However, only model $\mathrm{B}$, adjusting for traditional risk factors and medical history, remained statistically significant when comparing the fifth quintile with the first (HR 0.81; $95 \%$ CI 0.66, 0.99). As with intake of fatty fish, the test for trends was not statistically significant across quintiles in the adjusted analyses.

The associations between total marine $n$ - 3 PUFA and MI, modulated by restricted cubic splines, are presented in Fig. 2. For women, we found a trend towards an inverse association with MI, whereas for men the association was less strong. 
Table 1. Baseline characteristics of the cohort and cases with myocardial infarction*

(Medians and 10th and 90th percentiles (continuous variables) and numbers and percentages (categorical variables))

\begin{tabular}{|c|c|c|c|c|c|c|c|c|}
\hline \multirow[b]{3}{*}{ Variables } & \multicolumn{4}{|c|}{ Men } & \multicolumn{4}{|c|}{ Women } \\
\hline & \multicolumn{2}{|c|}{ Cohort (n 25913) } & \multicolumn{2}{|c|}{ Cases (n 2136) } & \multicolumn{2}{|c|}{ Cohort ( $n$ 28 991) } & \multicolumn{2}{|c|}{ Cases (n 892) } \\
\hline & Medians & $\begin{array}{l}\text { 10th; 90th } \\
\text { percentile }\end{array}$ & Medians & $\begin{array}{l}\text { 10th; 90th } \\
\text { percentile }\end{array}$ & Medians & $\begin{array}{l}\text { 10th; } 90 \text { th } \\
\text { percentile }\end{array}$ & Medians & $\begin{array}{l}\text { 10th; 90th } \\
\text { percentile }\end{array}$ \\
\hline Age (years) & 55.9 & $51 \cdot 2 ; 63.3$ & $57 \cdot 7$ & $51 \cdot 7 ; 63 \cdot 9$ & $56 \cdot 2$ & $51 \cdot 2 ; 63 \cdot 2$ & $59 \cdot 3$ & $52 \cdot 4 ; 64 \cdot 2$ \\
\hline Physical activity (h/week) & $2 \cdot 0$ & $0.0 ; 8.0$ & $2 \cdot 0$ & $0.0 ; 8.0$ & 2.5 & $0.0 ; 8.0$ & $2 \cdot 0$ & $0.0 ; 7.0$ \\
\hline BMI $\left(\mathrm{kg} / \mathrm{m}^{2}\right)$ & $26 \cdot 1$ & $22 \cdot 5 ; 31 \cdot 1$ & $26 \cdot 9$ & $23 \cdot 2 ; 32 \cdot 2$ & $24 \cdot 8$ & $20 \cdot 8 ; 31 \cdot 2$ & $25 \cdot 9$ & $20.9 ; 33.3$ \\
\hline Waist circumference $(\mathrm{cm})$ & 95.0 & $84.0 ; 108.5$ & $97 \cdot 0$ & $86.0 ; 112.0$ & $80 \cdot 0$ & $69.0 ; 97.0$ & $84 \cdot 0$ & $70 \cdot 0 ; 102 \cdot 0$ \\
\hline \multicolumn{9}{|l|}{ Smoking } \\
\hline $\begin{array}{l}n \\
\%\end{array}$ & & $\begin{array}{l}6772 \\
26 \cdot 1\end{array}$ & & $\begin{array}{l}389 \\
18 \cdot 2\end{array}$ & & $\begin{array}{c}12719 \\
43 \cdot 9\end{array}$ & & $\begin{array}{l}242 \\
27 \cdot 1\end{array}$ \\
\hline \multicolumn{9}{|l|}{ Former smoker } \\
\hline $\begin{array}{l}n \\
\%\end{array}$ & & $\begin{array}{c}8892 \\
34.3\end{array}$ & & $\begin{array}{l}631 \\
29.5\end{array}$ & & $\begin{array}{c}6797 \\
23.5\end{array}$ & & $\begin{array}{c}173 \\
19 \cdot 4\end{array}$ \\
\hline \multicolumn{9}{|l|}{$<15 \mathrm{~g} / \mathrm{d}$} \\
\hline$n$ & & 2744 & & 265 & & 4421 & & 198 \\
\hline$\%$ & & $10 \cdot 6$ & & $12 \cdot 4$ & & $15 \cdot 3$ & & $22 \cdot 2$ \\
\hline \multicolumn{9}{|l|}{$15-25 \mathrm{~g} / \mathrm{d}$} \\
\hline$n$ & & 4522 & & 509 & & 4305 & & 233 \\
\hline$\%$ & & 17.5 & & $23 \cdot 8$ & & 14.9 & & $26 \cdot 1$ \\
\hline \multicolumn{9}{|l|}{$>25 \mathrm{~g} / \mathrm{d}$} \\
\hline$n$ & & 2983 & & 342 & & 748 & & 46 \\
\hline$\%$ & & 11.5 & & $16 \cdot 0$ & & $2 \cdot 6$ & & $5 \cdot 2$ \\
\hline Educational level & & & & & & & & \\
\hline Basic school & & & & & & & & \\
\hline$n$ & & 8867 & & 923 & & 9043 & & 392 \\
\hline$\%$ & & $34 \cdot 2$ & & $43 \cdot 2$ & & $31 \cdot 2$ & & $44 \cdot 0$ \\
\hline Higher education, 1-3 years & & & & & & & & \\
\hline$n$ & & 10814 & & 796 & & 14556 & & 413 \\
\hline$\%$ & & $41 \cdot 7$ & & $37 \cdot 3$ & & $50 \cdot 2$ & & $46 \cdot 3$ \\
\hline Higher education, $>3$ years & & & & & & & & \\
\hline n & & 6232 & & 417 & & 5392 & & 87 \\
\hline$\%$ & & $24 \cdot 1$ & & 19.5 & & $18 \cdot 6$ & & 9.8 \\
\hline Menopausal status & & & & & & & & \\
\hline Postmenopausal & & & & & & & & \\
\hline$n$ & & - & & - & & 16975 & & 617 \\
\hline$\%$ & & - & & - & & 58.6 & & $69 \cdot 2$ \\
\hline Premenopausal & & & & & & & & \\
\hline$n$ & & - & & - & & 9022 & & 158 \\
\hline$\%$ & & - & & - & & $31 \cdot 1$ & & $17 \cdot 7$ \\
\hline Medical history & & & & & & & & \\
\hline Hypertension & & & & & & & & \\
\hline$n$ & & 3762 & & 472 & & 4984 & & 277 \\
\hline$\%$ & & 14.5 & & $22 \cdot 1$ & & $17 \cdot 2$ & & $31 \cdot 1$ \\
\hline Hypercholesterolaemia & & & & & & & & \\
\hline$n$ & & 2005 & & 253 & & 1762 & & $13 \cdot 1$ \\
\hline$\%$ & & $7 \cdot 7$ & & 11.8 & & $6 \cdot 1$ & & 117 \\
\hline Diabetes mellitus & & & & & & & & \\
\hline$n$ & & 677 & & 115 & & 435 & & 39 \\
\hline$\%$ & & $2 \cdot 6$ & & $5 \cdot 4$ & & 1.5 & & 4.4 \\
\hline Dietary variables $(\mathrm{g} / \mathrm{d})$ & & & & & & & & \\
\hline Fruits (excluding juice) & $117 \cdot 7$ & $27 \cdot 0 ; 324 \cdot 7$ & 111.0 & $25 \cdot 1 ; 313 \cdot 2$ & $172 \cdot 4$ & $49 \cdot 1 ; 417 \cdot 4$ & $160 \cdot 6$ & $37 \cdot 5 ; 399 \cdot 2$ \\
\hline $\begin{array}{l}\text { Vegetables (including juice, excluding } \\
\text { potatoes) }\end{array}$ & $151 \cdot 8$ & $62 \cdot 7 ; 291.4$ & $138 \cdot 8$ & $56 \cdot 7 ; 277 \cdot 5$ & 171.9 & $69.8 ; 329.4$ & $149 \cdot 2$ & $54.5 ; 318.3$ \\
\hline Alcohol & $19 \cdot 4$ & $3.6 ; 62.6$ & $18 \cdot 2$ & $2 \cdot 5 ; 62.7$ & $9 \cdot 3$ & $1.0 ; 34.5$ & 6.5 & $0.5 ; 31.9$ \\
\hline Total energy intake (MJ/d) & 9.9 & $7 \cdot 1 ; 13.6$ & $9 \cdot 8$ & $7 \cdot 1 ; 13.4$ & 8.0 & $5 \cdot 7 ; 11 \cdot 2$ & 7.9 & $5 \cdot 5 ; 11 \cdot 1$ \\
\hline Dietary fish intake & & & & & & & & \\
\hline Fatty fish & $15 \cdot 1$ & $4 \cdot 4 ; 37 \cdot 8$ & 14.9 & $4 \cdot 1 ; 37 \cdot 2$ & $12 \cdot 2$ & $3.6 ; 31.8$ & $11 \cdot 6$ & $2 \cdot 6 ; 31.0$ \\
\hline Lean fish & $24 \cdot 4$ & $10 \cdot 1 ; 50 \cdot 0$ & $24 \cdot 2$ & $10 \cdot 4 ; 51 \cdot 2$ & $21 \cdot 0$ & $9 \cdot 2 ; 42 \cdot 6$ & $20 \cdot 6$ & $8.5 ; 43.3$ \\
\hline Total fish & 41.7 & $17 \cdot 7 ; 81.5$ & 41.1 & $17 \cdot 3 ; 82 \cdot 3$ & 35.2 & $15 \cdot 1 ; 69 \cdot 8$ & 34.4 & $12 \cdot 8 ; 67.9$ \\
\hline EPA $(\mathrm{mg} / \mathrm{d})$ & $177 \cdot 3$ & $68 \cdot 7 ; 379 \cdot 1$ & $176 \cdot 5$ & $65 \cdot 7 ; 376 \cdot 6$ & $147 \cdot 5$ & $56 \cdot 1 ; 321 \cdot 7$ & $145 \cdot 2$ & $48 \cdot 3 ; 317 \cdot 7$ \\
\hline $\mathrm{DPA}(\mathrm{mg} / \mathrm{d})$ & $82 \cdot 0$ & $47 \cdot 3 ; 134 \cdot 5$ & $82 \cdot 7$ & $46 \cdot 5 ; 136 \cdot 5$ & $61 \cdot 8$ & $33.7 ; 105 \cdot 1$ & $62 \cdot 3$ & $31.6 ; 103.4$ \\
\hline $\mathrm{DHA}(\mathrm{mg} / \mathrm{d})$ & $430 \cdot 7$ & $189 \cdot 2 ; 866 \cdot 1$ & 428.8 & $179 \cdot 3 ; 855 \cdot 4$ & $358 \cdot 3$ & $151 \cdot 7 ; 726 \cdot 9$ & 354.5 & $132 \cdot 8 ; 705 \cdot 5$ \\
\hline Total marine $n-3$ PUFA $(\mathrm{mg} / \mathrm{d})$ & $692 \cdot 2$ & $313 \cdot 7 ; 1370 \cdot 1$ & $686 \cdot 6$ & $295 \cdot 2 ; 1355 \cdot 2$ & 569.2 & $248.8 ; 1148.3$ & 563.8 & $214 \cdot 8 ; 1115 \cdot 8$ \\
\hline Fish oil supplements & & & & & & & & \\
\hline$n$ & & 4116 & & 295 & & 5073 & & 295 \\
\hline$\%$ & & $15 \cdot 9$ & & $16 \cdot 4$ & & $17 \cdot 5$ & & $18 \cdot 8$ \\
\hline
\end{tabular}

DPA, docosapentaenoic acid.

* Comparison of baseline characteristics for the cohort as a whole (including both cases and non-cases) and cases with myocardial infarction separately. 
Fish consumption and myocardial infarction

\section{Fatal myocardial infarction}

As a secondary analysis, we evaluated the association of fish consumption and intake of marine $n-3$ PUFA with fatal MI (Table 4). After validation, 580 cases ( 424 men and 156 women) were categorised as having a fatal MI. Generally, the same tendency towards a negative association for fatty fish intake was observed; however, the CI were broader, and the associations were not statistically significant.

\section{Discussion}

In this Danish prospective cohort study conducted in middleaged men and women, we examined fish consumption and dietary intake of marine $n$-3 PUFA in relation to risk of MI. Dietary intake of fatty fish was inversely related to incident MI among men and women when comparing the highest and lowest quintiles, although the association only remained statistically significant for women after multivariate adjustments. For women, the same association was shown for total and individual marine $n$ - 3 PUFA with no material differences between EPA, DPA and DHA.

Moreover, two studies have been published previously, based on the Diet Cancer and Health cohort, examining the association of fish intake with adipose tissue marine $n$-3 PUFA content in participants with Acute Coronary Syndrome (ACS $)^{(6,7)}$. In the present study, the follow-up period was extended and the number of cases was larger providing considerably more strength to explore the associations in both men and women. Thus, Bjerregaard et al. ${ }^{(6)}$ found an inverse relationship between intake of fatty fish and ACS in men, suggesting a $32 \%$ lower risk from eating a moderate amount of fatty fish, whereas Joensen et al. ${ }^{(7)}$ demonstrated a lower and dose-dependent risk of ACS in men with a high content of marine $n-3$ PUFA in adipose tissue. Findings in women were inconsistent and non-significant in both studies ${ }^{(6,7)}$. In the present, substantially larger, study, we also demonstrated an inverse relationship in men, but the associations were generally attenuated during the longer follow-up period, and our results concerning fatty fish intake suggested a more modest relative risk of 0.88 when comparing the lowest and the highest quintiles in men. In contrast, our findings in women showed a consistent pattern compared with the earlier studies and suggested a $22 \%$ lower risk from high fish intake comparing the highest quintile with the lowest.

There is generally strong supportive evidence from epidemiological studies ${ }^{(5-12,15)}$ and secondary intervention trials ${ }^{(17-19)}$ that intake of fish and marine $n$-3 PUFA is associated with a lower risk of CHD and sudden cardiac death. Thus, in 2004 , He et al. conducted a meta-analysis of prospective cohort studies, suggesting that one fish serving/week is associated with a $15 \%$ lower risk of fatal CHD and 2-4 servings/week with a $23 \%$ lower risk. Today, both the American Heart Association ${ }^{(27)}$ and the European Society of Cardiology ${ }^{(28)}$ recommend a daily intake of marine $n-3$ PUFA of $1 \mathrm{~g} / \mathrm{d}$ for secondary prevention of $\mathrm{CHD}$

Recent clinical trials have questioned the beneficial effects of $n$-3 PUFA supplements ${ }^{(20-22)}$, and several reasons have been 
(a)

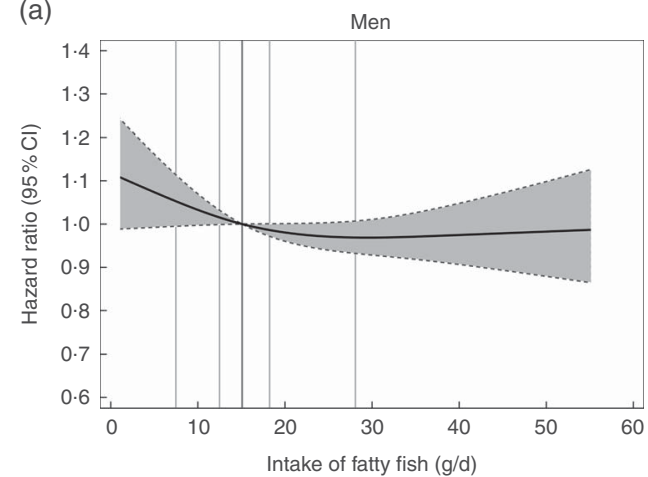

(b)

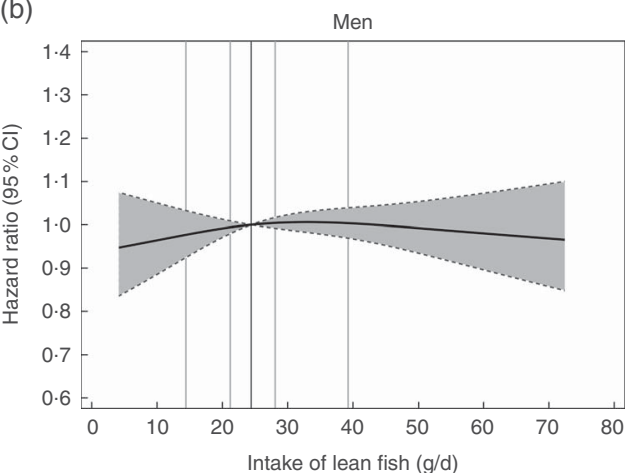

(c)

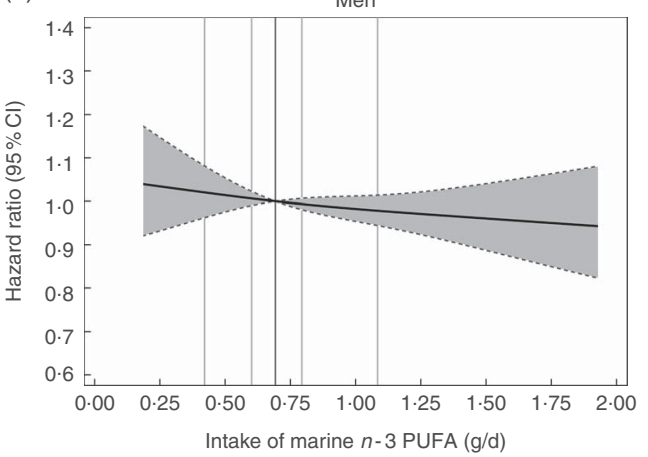

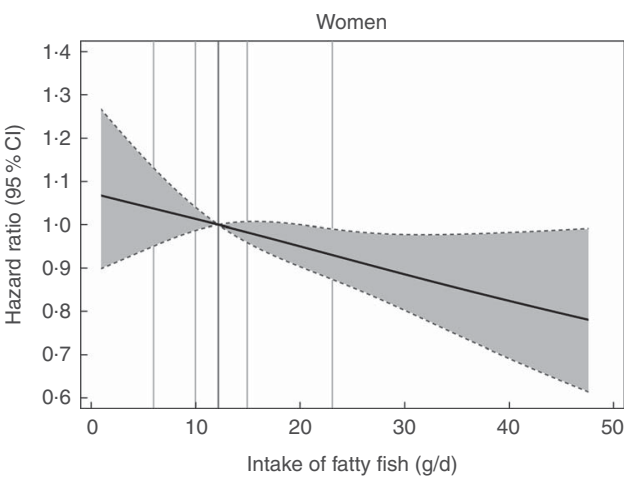
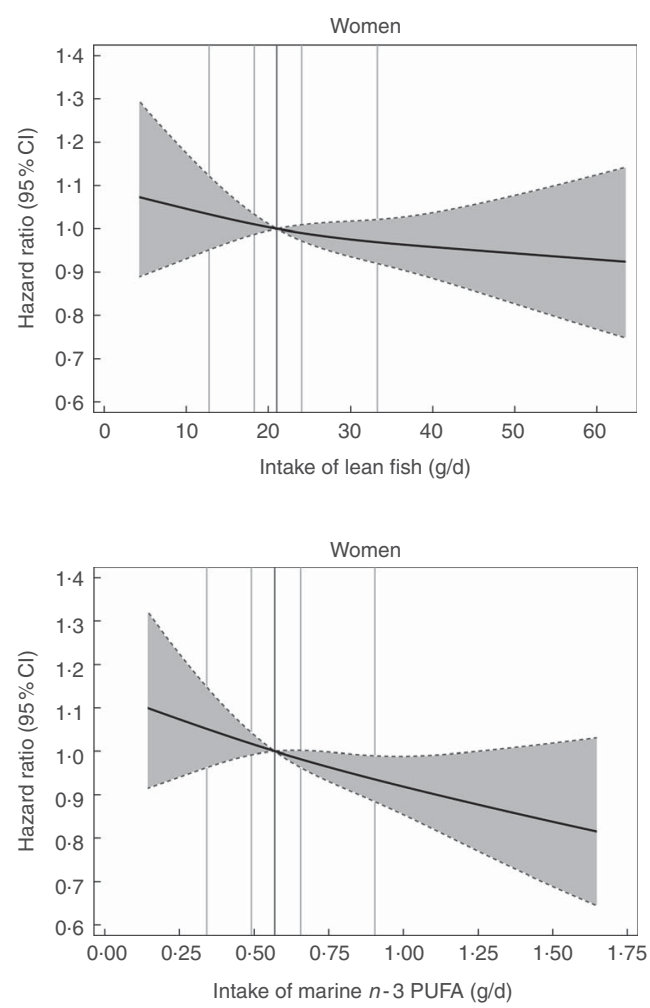

Fig. 2. Spline curves showing hazard ratios and $95 \% \mathrm{Cl}(\square)$ for the associations between (a) fatty fish, (b) lean fish, (c) total marine $n$-3 PUFA and incident myocardial infarction. Data presented for model B, adjusting for traditional risk factors and medical history, with the median dietary intake as reference. — Intake by quintiles.

proposed to explain these findings. First of all, methodological variations, different populations and different interventions might explain some of the differences. Notably, the recent trials were all conducted in populations with aggressive background therapy with antihypertensive, antiplatelet and lipid-lowering drugs, thereby diminishing the cardio-protective potential of $n$-3 PUFA. Furthermore, the effect of $n-3$ PUFA was mainly seen for fatal $\mathrm{CHD}$, whereas most recent trials have focused on non-fatal or composite end points for CHD, and finally the background dietary fish intake was generally higher in recent trials in accordance with rising public awareness of a healthy lifestyle ${ }^{(29)}$. In line with this, a recent meta-analysis reported a modest effect of marine $n$ - 3 PUFA on cardiovascular end points $^{(3)}$.

Several mechanisms have been suggested to explain the cardio-protective properties attributed to marine $n-3 \mathrm{PUFA}^{(2,4,30)}$.
These mechanisms may explain possible antithrombotic ${ }^{(19,31,32)}$ and/or antiarrhythmic ${ }^{(18,33,34)}$ effects of marine $n$-3 PUFA that may reduce the risk of $\mathrm{CHD}$. In addition, anti-inflammatory properties have been reported, possibly inferring a stabilising effect of marine $n$-3 PUFA on human atherosclerotic plaques ${ }^{(35,36)}$ that may decrease the risk of plaque rupture, and thus MI and CHD. Finally, marine $n-3$ PUFA also lower plasma TAG $^{(37)}$, increase serum adiponectin ${ }^{(38,39)}$ and may slightly reduce blood pressure $^{(2)}$. The mechanisms by which $n$-3 PUFA interact with the risk of $\mathrm{MI}$ in the present study are uncertain, but the analyses suggest that the content of marine $n$ - 3 PUFA is important, as the inverse relationship between fish intake and MI was observed for fatty fish and not lean fish. Furthermore, intake of fatty fish was highly correlated with intake of total and individual n-3 PUFA (EPA and DHA in particular). A few studies have examined the effect of individual marine $n-3$ PUFA, as most trials have 
Table 3. Association of myocardial infarction and calculated intakes of individual and total marine $n-3$ PUFA* (Hazard ratios (HR) and $95 \%$ confidence intervals)

\begin{tabular}{|c|c|c|c|c|c|c|c|c|c|c|c|c|c|c|c|c|c|}
\hline & \multicolumn{8}{|c|}{ Men } & & \multicolumn{8}{|c|}{ Women } \\
\hline & \multicolumn{2}{|c|}{ Model A1† } & \multicolumn{2}{|c|}{ Model A2‡ } & \multicolumn{2}{|c|}{ Model B§ } & \multicolumn{2}{|c|}{ Model C\|I } & & \multicolumn{2}{|c|}{ Model A1† } & \multicolumn{2}{|c|}{ Model A2‡ } & \multicolumn{2}{|c|}{ Model B§ } & \multicolumn{2}{|c|}{ Model C\|I } \\
\hline & $\mathrm{HR}$ & $95 \% \mathrm{Cl}$ & $\mathrm{HR}$ & $95 \% \mathrm{Cl}$ & $\mathrm{HR}$ & $95 \% \mathrm{Cl}$ & $\mathrm{HR}$ & $95 \% \mathrm{Cl}$ & & $\mathrm{HR}$ & $95 \% \mathrm{Cl}$ & $\mathrm{HR}$ & $95 \% \mathrm{Cl}$ & $\mathrm{HR}$ & $95 \% \mathrm{Cl}$ & $\mathrm{HR}$ & $95 \% \mathrm{Cl}$ \\
\hline EPA & & & & & & & & & EPA & & & & & & & & \\
\hline Q1 (0-99 mg) & 1 & & 1 & & 1 & & 1 & & Q1 (0-82 mg) & 1 & & 1 & & 1 & & 1 & \\
\hline Q2 (>99-151 mg) & 0.88 & $0.77,1.01$ & 0.90 & $0.79,1.03$ & 0.89 & $0.78,1.02$ & 0.90 & $0.79,1.03$ & Q2 (>82-125 mg) & 0.93 & $0.76,1.14$ & 1.00 & $0.81,1.22$ & 0.98 & $0.80,1.21$ & 1.01 & $0.82,1.24$ \\
\hline Q3 (>151-207 mg) & 0.93 & $0.82,1.06$ & 0.98 & $0.86,1.12$ & 0.97 & $0.85,1.11$ & 0.97 & $0.85,1.12$ & Q3 (>125-173 mg) & 0.74 & $0.60,0.92$ & 0.82 & $0.66,1.02$ & 0.80 & $0.65,1.00$ & 0.84 & $0.67,1.05$ \\
\hline Q4 (>207-294 mg) & 0.92 & $0.81,1.05$ & 0.99 & $0.87,1.13$ & 0.97 & $0.85,1.11$ & 0.98 & $0.86,1.13$ & 73-247 mg) & 1.00 & $0.82,1.22$ & 1.14 & $0.93,1.39$ & 1.11 & $0.91,1.35$ & 1.18 & $0.96,1.46$ \\
\hline Q5 (>294 mg) & 0.89 & $0.78,1.01$ & 0.93 & $0.81,1.07$ & 0.90 & $0.79,1.03$ & 0.92 & $0.80,1.07$ & Q5 $(>247 \mathrm{mg})$ & 0.75 & $0.61,0.92$ & 0.83 & $0.67,1.03$ & 0.79 & $0.64,0.97$ & 0.87 & $0.69,1.10$ \\
\hline$P_{\text {trend }}$ & \multicolumn{2}{|c|}{$P=0.20$} & \multicolumn{2}{|c|}{$P=0.76$} & \multicolumn{2}{|c|}{$P=0.42$} & \multicolumn{2}{|c|}{$P=0.69$} & $P_{\text {trend }}$ & \multicolumn{2}{|c|}{$P=0.04$} & \multicolumn{2}{|c|}{$P=0.36$} & \multicolumn{2}{|c|}{$P=0.14$} & \multicolumn{2}{|c|}{$P=0.73$} \\
\hline DPA & & & & & & & & & DPA & & & & & & & & \\
\hline Q1 (0-58 mg) & 1 & & 1 & & 1 & & 1 & & Q1 (0-42 mg) & 1 & & 1 & & 1 & & 1 & \\
\hline Q2 (>58-74 mg) & 0.87 & $0.76,1.00$ & 0.88 & $0.77,1.01$ & 0.88 & $0.77,1.01$ & 0.87 & $0.75,1.00$ & $2-56 \mathrm{mg})$ & 0.79 & $0.64,0.97$ & 0.83 & $0.68,1.02$ & 0.83 & $0.67,1.02$ & 0.84 & $0.68,1.04$ \\
\hline Q3 (>74-91 mg) & 0.98 & $0.86,1 \cdot 12$ & 0.98 & $0.86,1.12$ & 0.99 & $0.87,1.13$ & 0.97 & $0.84,1 \cdot 12$ & Q3 (>56-69 mg) & 0.77 & $0.63,0.94$ & 0.84 & $0.68,1.03$ & 0.83 & $0.67,1.02$ & 0.86 & $0.69,1.07$ \\
\hline Q4 (>91-113 mg) & 0.98 & $0.86,1.12$ & 0.98 & $0.85,1.12$ & 0.98 & $0.85,1.11$ & 0.96 & $0.82,1.11$ & Q4 (>69-88 mg) & 0.87 & $0.71,1.06$ & 0.92 & $0.76,1 \cdot 13$ & 0.91 & $0.74,1 \cdot 11$ & 0.95 & $0.76,1.20$ \\
\hline Q5 (>113 mg) & 0.97 & $0.85,1.11$ & 0.94 & $0.82,1.07$ & 0.94 & $0.82,1.07$ & 0.92 & $0.78,1.08$ & Q5 (>88 mg) & 0.80 & $0.65,0.98$ & & $0.67,1.00$ & 0.81 & $0.66,0.99$ & 0.84 & $0.66,1.08$ \\
\hline$P_{\text {trend }}$ & \multicolumn{2}{|c|}{$P=0.78$} & \multicolumn{2}{|c|}{$P=0.84$} & \multicolumn{2}{|c|}{$P=0.61$} & \multicolumn{2}{|c|}{$P=0.74$} & $P_{\text {trend }}$ & \multicolumn{2}{|c|}{$P=0.11$} & \multicolumn{2}{|c|}{$P=0.19$} & \multicolumn{2}{|c|}{$P=0 \cdot 13$} & & - 016 \\
\hline DHA & & & & & & & & & DHA & & & & & & & & \\
\hline Q1 (0-2 & 1 & & 1 & & 1 & & 1 & & Q1 (0-2 & 1 & & 1 & & 1 & & 1 & \\
\hline Q2 (>257-373 mg) & 0.91 & $0.80,1.05$ & 0.93 & $0.81,1.06$ & 0.93 & $0.81,1.06$ & 0.92 & $0.80,1.05$ & $-307 \mathrm{mg})$ & 0.81 & $0.66,0.99$ & 0.86 & $0.70,1.06$ & 0.87 & $0.71,1.07$ & 0.87 & $0.71,1.08$ \\
\hline Q3 (>373-496 mg) & 0.92 & $0.80,1.05$ & 0.95 & $0.83,1.08$ & 0.95 & $0.83,1.09$ & 0.94 & $0.82,1.07$ & Q3 (>307-413 mg) & 0.79 & $0.64,0.96$ & 0.87 & $0.70,1.07$ & 0.86 & $0.70,1.05$ & 0.89 & $0.72,1.10$ \\
\hline Q4 (>496-683 mg) & 0.96 & $0.84,1 \cdot 10$ & 1.01 & $0.88,1 \cdot 15$ & 1.00 & $0.87,1.14$ & 0.99 & $0.86,1 \cdot 14$ & $3-573 \mathrm{mg})$ & 0.88 & $0.72,1.07$ & 0.97 & $0.79,1 \cdot 18$ & 0.95 & $0.78,1 \cdot 16$ & 0.99 & $0.80,1.23$ \\
\hline Q5 (>683 mg) & 0.90 & $0.79,1.03$ & 0.92 & $0.80,1.05$ & 0.90 & $0.78,1.03$ & 0.90 & $0.77,1.05$ & Q5 (>573 mg) & 0.75 & $0.61,0.93$ & 0.82 & $0.66,1.01$ & 0.79 & $0.64,0.97$ & 0.85 & $0.67,1.07$ \\
\hline$P_{\text {trend }}$ & & $=0.29$ & & $=0.56$ & & $=0.34$ & & $=0.44$ & $P_{\text {trend }}$ & & - & & $=0.22$ & & $=0.09$ & & $=0.49$ \\
\hline Total marine $n-3$ PUFA & & & & & & & & & Total marine $n-3$ PUFA & & & & & & & & \\
\hline Q1 (0-421 mg) & 1 & & 1 & & 1 & & 1 & & Q1 (0-341 mg) & 1 & & 1 & & 1 & & 1 & \\
\hline Q2 (>421-602 mg) & 0.94 & $0.82,1.07$ & 0.95 & $0.83,1.09$ & 0.95 & $0.83,1.08$ & 0.94 & $0.82,1.08$ & Q2 (>341-491 mg) & 0.84 & $0.68,1.03$ & 0.90 & $0.74,1 \cdot 11$ & 0.91 & $0.74,1 \cdot 11$ & 0.92 & $0.75,1 \cdot 13$ \\
\hline Q3 (>602-793 mg) & 0.92 & $0.80,1.05$ & 0.95 & $0.83,1.09$ & 0.96 & $0.84,1.09$ & 0.94 & $0.82,1.08$ & Q3 (>491-656 mg) & 0.78 & $0.63,0.96$ & 0.86 & $0.70,1.06$ & 0.85 & $0.69,1.04$ & 0.88 & $0.71,1 \cdot 10$ \\
\hline Q4 (>793-1083 mg) & 0.95 & $0.83,1.09$ & 1.00 & $0.87,1.14$ & 0.99 & $0.86,1.13$ & 0.98 & $0.85,1.13$ & Q4 (>656-904 mg) & 0.88 & $0.72,1.07$ & 0.97 & $0.80,1 \cdot 19$ & 0.95 & $0.78,1 \cdot 17$ & 1.01 & $0.81,1.25$ \\
\hline Q5 (>1083 mg) & 0.92 & $0.80,1.05$ & 0.94 & $0.82,1.07$ & 0.91 & $0.80,1.04$ & 0.92 & $0.79,1.07$ & Q5 (>904 mg) & 0.77 & $0.63,0.95$ & 0.84 & $0.69,1.04$ & 0.81 & $0.66,0.99$ & 0.88 & $0.69,1.11$ \\
\hline$P_{\text {trend }}$ & & $P=0.29$ & & $P=0.60$ & & $=0.36$ & & $=0.49$ & $P_{\text {trend }}$ & & $=0.04$ & & $=0.25$ & & $=0.11$ & & $=0.56$ \\
\hline
\end{tabular}

Q, quintiles; DPA, docosapentaenoic acid.

* $P$ values test the hypothesis of no trend across quintiles.

$\dagger$ Model A1: crude analysis.

‡ Model A2: adjusted for traditional risk factors including smoking, BMI, waist circumference, physical activity, alcohol intake, educational level and menopausal status (women).

$\S$ Model B: adjusted as model A2 with additional covariates: history of diabetes mellitus, hypertension and hypercholesterolaemia.

॥ Model C: adjusted as model A2 with additional dietary covariates: total energy intake, total intake of SFA, MUFA and PUFA (excl. marine $n-3$ PUFA) and dietary fibre. 
Table 4. Association of dietary fish consumption and intake of marine $n-3$ PUFA with fatal myocardial infarction (Hazard ratios (HR) and $95 \%$ confidence intervals)

\begin{tabular}{|c|c|c|c|c|c|c|c|c|c|}
\hline & \multicolumn{4}{|c|}{ Men } & & \multicolumn{4}{|c|}{ Women } \\
\hline & Model A1† & Model A2 $\ddagger$ & Model B§ & Model C\| & & Model A1† & Model A2 $\ddagger$ & Model B§ & Model C\| \\
\hline & $95 \% \mathrm{Cl}$ & $95 \% \mathrm{Cl}$ & $95 \% \mathrm{Cl}$ & $95 \% \mathrm{Cl}$ & & $95 \% \mathrm{Cl}$ & $95 \% \mathrm{Cl}$ & $95 \% \mathrm{Cl}$ & $95 \% \mathrm{Cl}$ \\
\hline \multicolumn{5}{|l|}{ Intake of fatty fish } & \multicolumn{5}{|l|}{ Intake of fatty fish } \\
\hline Q1 $(0-8 \mathrm{~g})$ & 1 & 1 & 1 & 1 & Q1 $(0-6 \mathrm{~g})$ & 1 & 1 & 1 & 1 \\
\hline Q2 (>8-13g) & $0.750 .56,1.01$ & $0.77 \quad 0.57,1.04$ & $0.76 \quad 0.57,1.03$ & $0.790 .59,1.07$ & Q2 (>6-10 g) & $1.040 .66,1.63$ & $1.15 \quad 0.73,1.82$ & $1.150 .73,1.81$ & $1.18 \quad 0.75,1.87$ \\
\hline Q3 $(>13-18 \mathrm{~g})$ & $0.740 .55,1.00$ & $0.80 \quad 0.59,1.06$ & $0.80 \quad 0.60,1.08$ & $0.820 .60,1.10$ & Q3 $(>10-15 \mathrm{~g})$ & $0.920 .58,1.46$ & $1.060 .67,1.70$ & $1.040 .65,1.65$ & $1.10 \quad 0.69,1.78$ \\
\hline Q4 $(>18-28 \mathrm{~g})$ & $0.76 \quad 0.57,1.02$ & $0.850 .63,1.14$ & $0.85 \quad 0.63,1.14$ & $0.88 \quad 0.65,1.19$ & Q4 (>15-23 g) & $0.49 \quad 0.28,0.85$ & $0.60 \quad 0.34,1.05$ & $0.58 \quad 0.33,1.02$ & $0.620 .35,1.09$ \\
\hline Q5 $(>28 \mathrm{~g})$ & $0.78 \quad 0.58,1.04$ & $0.86 \quad 0.64,1.15$ & $0.810 .61,1.09$ & $0.890 .65,1.21$ & Q5 $(>23 \mathrm{~g})$ & $0.750 .47,1.21$ & $0.94 \quad 0.58,1.52$ & $0.870 .53,1.40$ & $0.940 .56,1.56$ \\
\hline$P_{\text {trend }}$ & $P=0.13$ & $P=0.50$ & $P=0.33$ & $P=0.66$ & $P_{\text {trend }}$ & $P=0.03$ & $P=0.22$ & $P=0.12$ & $P=0.25$ \\
\hline \multicolumn{5}{|l|}{ Intake of lean fish } & \multicolumn{5}{|l|}{ Intake of lean fish } \\
\hline Q1 $(0-8 \mathrm{~g})$ & 1 & 1 & 1 & 1 & Q1 $(0-6 \mathrm{~g})$ & 1 & 1 & 1 & 1 \\
\hline Q2 (>8-13g) & $0.90 \quad 0.65,1.24$ & $0.950 .69,1.31$ & $0.97 \quad 0.70,1.34$ & $0.990 .72,1.37$ & Q2 (>6-10 g) & $0.78 \quad 0.47,1.31$ & $0.850 .51,1.42$ & $0.860 .51,1.44$ & $0.890 .53,1.50$ \\
\hline Q3 $(>13-18 \mathrm{~g})$ & $1.110 .82,1.50$ & $1.170 .87,1.59$ & $1.160 .86,1.58$ & $1.26 \quad 0.92,1.71$ & Q3 $(>10-15 \mathrm{~g})$ & $1.07 \quad 0.67,1.71$ & $1.18 \quad 0.74,1.89$ & $1.170 .73,1.88$ & $1.250 .77,2.03$ \\
\hline Q4 (>18-28 g) & $1.090 .80,1.48$ & $1.180 .87,1.60$ & $1.17 \quad 0.86,1.58$ & $1.270 .93,1.74$ & Q4 (>15-23 g) & $0.91 \quad 0.56,1.48$ & $1.040 .64,1.70$ & $1.030 .63,1.68$ & $1.120 .67,1.86$ \\
\hline Q5 $(>28 \mathrm{~g})$ & $1.190 .88,1.60$ & $1.230 .91,1.66$ & $1.180 .88,1.60$ & $1.34 \quad 0.97,1.84$ & Q5 $(>23 \mathrm{~g})$ & $0.95 \quad 0.59,1.54$ & $1.050 .65,1.70$ & $1.03 \quad 0.64,1.68$ & $1.120 .66,1.90$ \\
\hline$P_{\text {trend }}$ & $P=0.12$ & $P=0.07$ & $P=0.14$ & $P=0.03$ & $P_{\text {trend }}$ & $P=0.95$ & $P=0.62$ & $P=0.70$ & $P=0.46$ \\
\hline \multicolumn{5}{|l|}{ Total marine $n$-3 PUFA intake } & \multicolumn{5}{|l|}{ Total marine $n-3$ PUFA intake } \\
\hline Q1 (0-421 mg) & 1 & 1 & 1 & 1 & Q1 (0-341 mg) & 1 & 1 & 1 & 1 \\
\hline Q2 (>421-602 mg) & $0.760 .55,1.04$ & $0.770 .56,1.05$ & $0.750 .55,1.03$ & $0.760 .56,1.05$ & Q2 (>341-491 mg) & $1.090 .69,1.72$ & $1.200 .76,1.90$ & $1.190 .75,1.89$ & $1.250 .78,2.00$ \\
\hline Q3 (>602-793 mg) & $0.96 \quad 0.72,0.29$ & $1.00 \quad 0.74,1.34$ & $1.010 .75,1.35$ & $1.00 \quad 0.73,1.35$ & Q3 (>491-656 mg) & $0.820 .50,1.33$ & $0.920 .56,1.50$ & $0.90 \quad 0.55,1.47$ & $0.940 .56,1.57$ \\
\hline Q4 (>793-1083 mg) & $0.93 \quad 0.69,1.25$ & $0.98 \quad 0.73,1.32$ & $0.960 .72,1.30$ & $0.990 .72,1.35$ & Q4 (>656-904 mg) & $0.75 \quad 0.45,1.23$ & $0.86 \quad 0.52,1.43$ & $0.84 \quad 0.51,1.39$ & $0.90 \quad 0.52,1.54$ \\
\hline Q5 (>1083 mg) & $0.940 .70,1.26$ & $0.95 \quad 0.71,1.28$ & $0.90 \quad 0.67,1.21$ & $0.99 \quad 0.71,1.37$ & Q5 (>904 mg) & $0.750 .46,1.23$ & $0.850 .52,1.39$ & $0.810 .49,1.33$ & $0.790 .44,1.41$ \\
\hline$P_{\text {trend }}$ & $P=0.86$ & $P=0.72$ & $P=0.97$ & $P=0.58$ & $P_{\text {trend }}$ & $P=0.09$ & $P=0.24$ & $P=0.17$ & $P=0.22$ \\
\hline \multicolumn{10}{|c|}{$\begin{array}{l}Q, \text { quintiles. } \\
{ }^{*} P \text { values test the hypothesis of no trend across quintiles. }\end{array}$} \\
\hline
\end{tabular}


examined an intervention consisting of a combination of EPA and DHA, as these are the major marine $n$ - 3 PUFA found in seafood and fish oil. However, a large intervention trial in a Japanese population used a supplement of purified EPA and produced a significant reduction in major cardiovascular events, suggesting anti-atherosclerotic effects of EPA independently of other marine $n-3$ PUFA $^{(19)}$. Accordingly, important differential effects have been suggested between the major $n$-3 PUFA, but more studies on individual $n$-3 PUFA are warranted ${ }^{(40)}$. In the present study, we showed that EPA, DPA and DHA were similarly related to MI, but the study design did not allow for further investigation into the mechanistic aspects of individual $n$-3 PUFA.

\section{Strengths and limitations}

This study was based on a large prospective cohort study and holds the advantages of the prospective design.

The dietary intake of fish and marine $n$ - 3 PUFA was evaluated in a detailed manner. First, we assessed fish intake using detailed semi-quantitative FFQ including twenty-six questions concerning fish intake. This allowed us to differentiate by types of fish, thereby grouping fish consumption into fatty and lean fish depending on the content of marine $n-3$ PUFA. Second, we calculated the intake of major marine $n-3$ PUFA (EPA, DPA and DHA), to assess these fatty acids individually.

There was a limited loss to follow-up, and the assessment of outcome data was thorough and complete. All outcome data from the National Patient Registry or Causes of Death Registry were either examined from medical records or from a complete list of diagnoses and medical procedure codes, ensuring high validity of cases.

Adjustment for potential confounding was applied in different steps allowing for a more detailed interpretation of the results. In model A2, we adjusted for traditional risk factors for MI, which has a clear and straightforward interpretation. In model $\mathrm{B}$, we applied a second layer of variables adjusting for medical history. These variables are normally considered traditional risk factors for MI and potential confounders, but at the same time may represent intermediate steps in causal pathways by which $n$-3 PUFA affect the risk of MI. In our opinion, model B represents the most complete analysis including important covariates, and the risk of introducing bias by including intermediate variables is outweighed by the risk of residual confounding by not including these variables. This is, however, open for discussion. Finally, we included dietary variables in addition to the traditional risk factors from model A2 (model C). This model implies adjustment for potential confounding from other aspects of the diet, but it may also introduce dietary patterns that are less comparable with the ordinary dietary pattern. Generally, the differences in measures of association between models were moderate or small, suggesting that confounding was not a major concern in this study.

This study also has certain limitations. Although age was evenly distributed among men and women, there was a markedly higher proportion of male cases and there were relatively few female cases, which made the CI wider for measures of association when analysing data in women, particularly fatal cases. The median follow-up period was $17 \cdot 0$ years, and dietary measures were not assessed during the study period. A long follow-up period allowed us to accumulate more outcome events, but the participants might have changed their diet over time. Furthermore, changes in standard medical care and general changes in lifestyle and public awareness of disease prevention might have influenced the participants' risk profile. To address this issue, we performed supplementary analysis by stratifying date of birth and testing for interaction from date of birth. No significant interaction was detected, and stratification did not affect the measures of association. Another concern when using FFQ is the chance of measurement error and various sources of bias, and evaluating biomarkers of marine $n-3$ PUFA intake would add strength to the dietary data. In this article we do not present data on biomarkers of $n-3$ PUFA, but our group recently published an article investigating the association between adipose tissue content of marine $n$-3 PUFA and MI, supporting the findings of this dietary study $^{(41)}$

\section{Conclusions}

In this prospective cohort study, we found a high intake of fatty fish to be inversely related to incident MI in both men and women when comparing the highest and lowest quintiles. However, a clear dose-response relationship could not be established, and the test for trends across quintiles was not statistically significant in the adjusted analyses. Lean fish was not associated with MI. This study supports the current view that consumption of fatty fish may protect against MI.

\section{Acknowledgements}

This study was supported by a research grant from The Danish Heart Association, Copenhagen, Denmark, and the Hertha Christensen Foundation, Aalborg, Denmark. The Diet, Cancer and Health study was funded by the Danish Cancer Society. The sponsors neither had influence on the design and conduct of the study nor the writing of the manuscript.

All the authors contributed to the conception and planning of the study. A. G. and S. L.-C. were responsible for the statistical analysis of the data, and all authors were involved in interpretation of the data and writing of the manuscript. A. G. wrote the first draft of the manuscript.

The authors declare that there are no conflicts of interest regarding the present study.

\section{Supplementary material}

For supplementary material/s referred to in this article, please visit http://dx.doi.org/10.1017/S000711451600180X

\section{References}

1. He K, Song Y, Daviglus ML, et al. (2004) Accumulated evidence on fish consumption and coronary heart disease mortality: a meta-analysis of cohort studies. Circulation 109, $2705-2711$.

2. De Caterina R (2011) n-3 Fatty acids in cardiovascular disease. $N$ Engl J Med 364, 2439-2450. 
3. Rizos EC, Ntzani EE, Bika E, et al. (2012) Association between omega-3 fatty acid supplementation and risk of major cardiovascular disease events: a systematic review and metaanalysis. JAMA 308, 1024-1033.

4. Saravanan P, Davidson NC, Schmidt EB, et al. (2010) Cardiovascular effects of marine omega-3 fatty acids. Lancet 375, 540-550.

5. Lemaitre RN, King IB, Mozaffarian D, et al. (2003) n-3 Polyunsaturated fatty acids, fatal ischemic heart disease, and nonfatal myocardial infarction in older adults: the Cardiovascular Health Study. Am J Clin Nutr 77, 319-325.

6. Bjerregaard LJ, Joensen AM, Dethlefsen C, et al. (2010) Fish intake and acute coronary syndrome. Eur Heart $J \mathbf{3 1}$, 29-34.

7. Joensen AM, Overvad K, Dethlefsen C, et al. (2011) Marine $n-3$ polyunsaturated fatty acids in adipose tissue and the risk of acute coronary syndrome. Circulation $\mathbf{1 2 4}$, $1232-1238$.

8. Miyagawa N, Miura K, Okuda N, et al. (2014) Long-chain $n-3$ polyunsaturated fatty acids intake and cardiovascular disease mortality risk in Japanese: a 24-year follow-up of NIPPON DATA80. Atherosclerosis 232, 384-389.

9. Daviglus ML, Stamler J, Orencia AJ, et al. (1997) Fish consumption and the 30-year risk of fatal myocardial infarction. N Engl J Med 336, 1046-1053.

10. de Goede J, Geleijnse JM, Boer JMA, et al. (2010) Marine (n-3) fatty acids, fish consumption, and the 10-year risk of fatal and nonfatal coronary heart disease in a large population of Dutch adults with low fish intake. J Nutr 140, 1023-1028.

11. Hu FB, Bronner L, Willett WC, et al. (2002) Fish and omega-3 fatty acid intake and risk of coronary heart disease in women. JAMA 287, 1815-1821.

12. Streppel MT, Ocké MC, Boshuizen HC, et al. (2008) Long-term fish consumption and $n-3$ fatty acid intake in relation to (sudden) coronary heart disease death: the Zutphen study. Eur Heart J 29, 2024-2030.

13. Oomen CM, Feskens EJ, Räsänen L, et al. (2000) Fish consumption and coronary heart disease mortality in Finland, Italy, and The Netherlands. Am J Epidemiol 151, 999-1006.

14. Yuan JM, Ross RK, Gao YT, et al. (2001) Fish and shellfish consumption in relation to death from myocardial infarction among men in Shanghai, China. Am J Epidemiol 154, 809-816.

15. Amiano P, Machón M, Dorronsoro M, et al. (2013) Intake of total omega-3 fatty acids, eicosapentaenoic acid and docosahexaenoic acid and risk of coronary heart disease in the Spanish EPIC cohort study. Nutr Metab Cardiovasc Dis 24, 321-327.

16. Ascherio A, Rimm EB, Stampfer MJ, et al. (1995) Dietary intake of marine $n-3$ fatty acids, fish intake, and the risk of coronary disease among men. $N$ Engl J Med 332, 977-982.

17. Burr ML, Fehily AM, Gilbert JF, et al. (1989) Effects of changes in fat, fish, and fibre intakes on death and myocardial reinfarction: diet and reinfarction trial (DART). Lancet 344, 757-761.

18. Gruppo Italiano per lo Studio della Sopravvivenza nell'Infarto miocardico (1999) Dietary supplementation with n-3 polyunsaturated fatty acids and vitamin $\mathrm{E}$ after myocardial infarction: results of the GISSI-Prevenzione trial. Lancet 354, $447-455$.

19. Saito Y, Yokoyama M, Origasa H, et al. (2008) Effects of EPA on coronary artery disease in hypercholesterolemic patients with multiple risk factors: sub-analysis of primary prevention cases from the Japan EPA Lipid Intervention Study (JELIS). Atherosclerosis 200, 135-140.
20. Kromhout D, Giltay EJ \& Geleijnse JM (2010) n-3 Fatty acids and cardiovascular events after myocardial infarction. $N$ Engl J Med 363, 2015-2026.

21. Rauch B, Schiele R, Schneider S, et al. (2010) OMEGA, a randomized, placebo-controlled trial to test the effect of highly purified omega-3 fatty acids on top of modern guidelineadjusted therapy after myocardial infarction. Circulation 122, 2152-2159.

22. Galan P, Kesse-Guyot E, Czernichow S, et al. (2010) Effects of B vitamins and omega 3 fatty acids on cardiovascular diseases: a randomised placebo controlled trial. BMJ 341, c6273.

23. Tjønneland A, Olsen A, Boll K, et al. (2007) Study design, exposure variables, and socioeconomic determinants of participation in Diet, Cancer and Health: a population-based prospective cohort study of 57053 men and women in Denmark. Scand J Public Health 35, 432-441.

24. Tjønneland A, Overvad K, Haraldsdóttir J, et al. (1991) Validation of a semiquantitative food frequency questionnaire developed in Denmark. Int J Epidemiol 20, 906-912.

25. Andersen TF, Madsen M, Jørgensen J, et al. (1999) The Danish National Hospital Register. A valuable source of data for modern health sciences. Dan Med Bull 46, 263-268.

26. Joensen AM, Jensen MK, Overvad K, et al. (2009) Predictive values of acute coronary syndrome discharge diagnoses differed in the Danish National Patient Registry. J Clin Epidemiol 62, 188-194.

27. Kris-Etherton PM, Harris WS \& Appel LJ (2003) Omega-3 fatty acids and cardiovascular disease: new recommendations from the American Heart Association. Arterioscler Thromb Vasc Biol 23, 151-152.

28. Catapano AL, Reiner Z, De Backer G, et al. (2011) ESC/EAS Guidelines for the management of dyslipidaemias The Task Force for the management of dyslipidaemias of the European Society of Cardiology (ESC) and the European Atherosclerosis Society (EAS). Atherosclerosis 217, 3-46.

29. Wu JHY \& Mozaffarian D (2014) $\omega-3$ fatty acids, atherosclerosis progression and cardiovascular outcomes in recent trials: new pieces in a complex puzzle. Heart 100, 530-533.

30. Mozaffarian D \& Wu JHY (2011) Omega-3 fatty acids and cardiovascular disease: effects on risk factors, molecular pathways, and clinical events. J Am Coll Cardiol 58, 2047-2067.

31. Rodriguez BL, Sharp DS, Abbott RD, et al. (1996) Fish intake may limit the increase in risk of coronary heart disease morbidity and mortality among heavy smokers. The Honolulu Heart Program. Circulation 94, 952-956.

32. Eshak ES, Iso H, Yamagishi K, et al. (2014) Modification of the excess risk of coronary heart disease due to smoking by seafood/fish intake. Am J Epidemiol 179, 1173-1181.

33. London B, Albert C, Anderson ME, et al. (2007) Omega-3 fatty acids and cardiac arrhythmias: prior studies and recommendations for future research: a report from the National Heart, Lung, and Blood Institute and Office Of Dietary Supplements Omega-3 Fatty Acids and their Role in Cardiac Arrhythmogenesis Workshop. Circulation 116, 320-335.

34. Christensen JH (2003) n-3 Fatty acids and the risk of sudden cardiac death. Emphasis on heart rate variability. Dan Med Bull 50, 347-367.

35. Thies F, Garry JMC, Yaqoob P, et al. (2003) Association of $n-3$ polyunsaturated fatty acids with stability of atherosclerotic plaques: a randomised controlled trial. Lancet 361, 477-485.

36. Calder PC (2012) The role of marine omega-3 (n-3) fatty acids in inflammatory processes, atherosclerosis and plaque stability. Mol Nutr Food Res 56, 1073-1080. 
37. Eslick GD, Howe PRC, Smith C, et al. (2009) Benefits of fish oil supplementation in hyperlipidemia: a systematic review and meta-analysis. Int J Cardiol 136, 4-16.

38. Gammelmark A, Madsen T, Varming K, et al. (2012) Low-dose fish oil supplementation increases serum adiponectin without affecting inflammatory markers in overweight subjects. Nutr Res 32, 15-23.

39. Wu JHY, Cahill LE \& Mozaffarian D (2013) Effect of fish oil on circulating adiponectin: a systematic review and meta-analysis of randomized controlled trials. J Clin Endocrinol Metab 98 2451-2459.

40. Mozaffarian D \& Wu JHY (2012) (n-3) Fatty acids and cardiovascular health: are effects of EPA and DHA shared or complementary? J Nutr 142, 614-625.

41. Gammelmark A, Nielsen MS, Bork C, et al. (2016) Adipose tissue content of marine $n-3$ polyunsaturated fatty acids is inversely associated with myocardial infarction. $J$ Am Coll Cardiol 67, 1008-1009. 